\title{
Understanding specific and nonspecific toxicities: a requirement for the development of dendrimer-based pharmaceuticals
}

\author{
Daniel Q. McNerny, ${ }^{1 *}$ Pascale R. Leroueil ${ }^{2}$ and James R. Baker ${ }^{3}$
}

\begin{abstract}
Dendrimer conjugates for pharmaceutical development are capable of enhancing the local delivery of cytotoxic drugs. The ability to conjugate different targeting ligands to the dendrimer allows for the cytotoxic drug to be focused at the intended target cell while minimizing collateral damage in normal cells. Dendrimers offer several advantages over other polymer conjugates by creating a better defined, more monodisperse therapeutic scaffold. Toxicity from the dendrimer, targeted and nonspecific, is not only dependent upon the number of targeting and therapeutic ligands conjugated, but can be influenced by the repeating building blocks that grow the dendrimer, the dendrimer generation, as well as the surface termination. @ 2010 John Wiley \& Sons, Inc. WIREs Nanomed Nanobiotechnol 20102 249-259
\end{abstract}

$\mathrm{T}$ he narrow therapeutic index of many cytotoxic therapeutics, including doxorubicin, vincristine, cyclophophamide, and paclitaxel, often limits their effectiveness as they must be delivered in suboptimal dosages to prevent side effects in the patient. ${ }^{1}$ To remedy this problem, targeted scaffolds can be used to deliver the drug the desired location in an increased, local concentration. As a result, the drug is effective only where it is needed and the undesired side toxicities are diminished. Examples of drug-targeting systems include nanoparticles, liposomes, micelles, linear polymers, branched polymers, and dendrimers. ${ }^{2}$

Dendrimer-based platforms have achieved attention for use in pharmaceutical applications. ${ }^{3-13}$ Similar to other polymeric carriers, dendrimers can be synthesized to avoid structural toxicity and immunogenicity. However, the unique branched structure of

*Correspondence to: dmcnerny@umich.com

${ }^{1}$ Department of Chemical Engineering, Michigan Nanotechnology Institute for Medicine and Biological Sciences

${ }^{2}$ Michigan Nanotechnology Institute for Medicine and Biological Sciences

${ }^{3}$ Department of Internal Medicine, Michigan Nanotechnology Institute for Medicine and Biological Sciences, University of Michigan, Ann Arbor, MI 48109, USA

DOI: $10.1002 /$ wnan.79 the dendrimer allows for the platform to overcome several significant challenges faced in the development of other polymeric carriers. Many polymers are highly heterogeneous, making characterization and batch reproducibility inherently difficult. Therapeutics with multiple drug or imaging moieties conjugated to a carry are heterogeneous populations that become more disperse as more functionalities are added. These problems often lead to unintended variations in biological activity, because the structural platform is not well understood and is difficult to reproduce. In contrast, the controlled synthesis and growth of dendrimers results in exceptionally low degrees of dispersity [polydispersity index $(\mathrm{PDI})<1.1$ ] $^{14,15}$ with well-defined numbers of terminal groups for the conjugation of functional molecules, allowing for improved reproducibility. The dendrimer's ability to mimic the size, solubility, and shape of human proteins makes the technology an ideal choice for many therapeutic and diagnostic applications. Being 1-10 nanometers in size enables dendrimers to efficiently diffuse across the vascular endothelium, internalize into cells, and be rapid cleared by the kidneys. ${ }^{16,17}$ This helps to avoid long-term toxicities and reduces the need for a rapidly degradable platform. The availability of multiple reactive surface groups enables the dendrimer 
to carry a higher payload of functional molecules, enhancing targeted toxicity.

Dendrimers have been produced or are under commercial development for several biomedical applications. A topical, polylysine dendrimer-based microbicide, VivaGel ${ }^{\mathrm{TM}}$, is being developed by Starpharma for the prevention of HIV transmission and other sexually transmitted diseases. SuperFect ${ }^{\circledR}$ is a dendrimerbased material used for gene transfection. Dendrimers have also been developed as diagnostic tools. Gadomer-17, a polylysine dendrimer functionalized with gadolinium chelates, was under development as a magnetic resonance imaging (MRI) contrast agent. Stratus ${ }^{\circledR}$ CS acts as a biosensor for cardiac markers in an effort to rapidly diagnosis heart attacks.

In recognition of these varied structures and applications, one must be careful not to generalize dendrimer biological activity and toxicity. The biocompatibility, solubility, and other characteristics of dendrimers are all highly dependent upon the chemical backbone of the dendrimer as well as the surface termination. These structural considerations must be evaluated when determining if a particular dendrimer is safe for pharmaceutical use. This review will detail the factors contributing to dendrimer biocompatibility and define any trends associated with structure. We will first briefly discuss dendrimer chemistry and outline which chemical backbones have been successfully used for biological applications. We will then detail targeted delivery of dendrimer scaffolds, which allows for enhanced specific toxicity. This will involve a discussion of the mechanisms involved in targeting as well as the mechanisms involved in the biological confirmation of the activity. Finally, we will address nonspecific toxicities related to dendrimer platforms and the means to minimize these effects.

\section{DENDRIMER CHEMISTRY}

Dendrimers are defined by their core-shell structure (Figure 1), where the dendrimer approximately doubles in size and number of functional surface groups with each additional shell (or generation) added to the core. Shells are synthesized by alternating monomer reactions by means reviewed extensively elsewhere. ${ }^{12,18}$ Specialized dendrimer backbones can be synthesized by varying the monomer units. The biological properties of the dendrimer are largely influenced by the chemical backbone and surface termination.

For a dendrimer to be an appropriate vehicle for drug delivery in vivo, they must be nontoxic, nonimmunogenic, and be capable of targeting and reaching specific locations by crossing the appropriate barriers

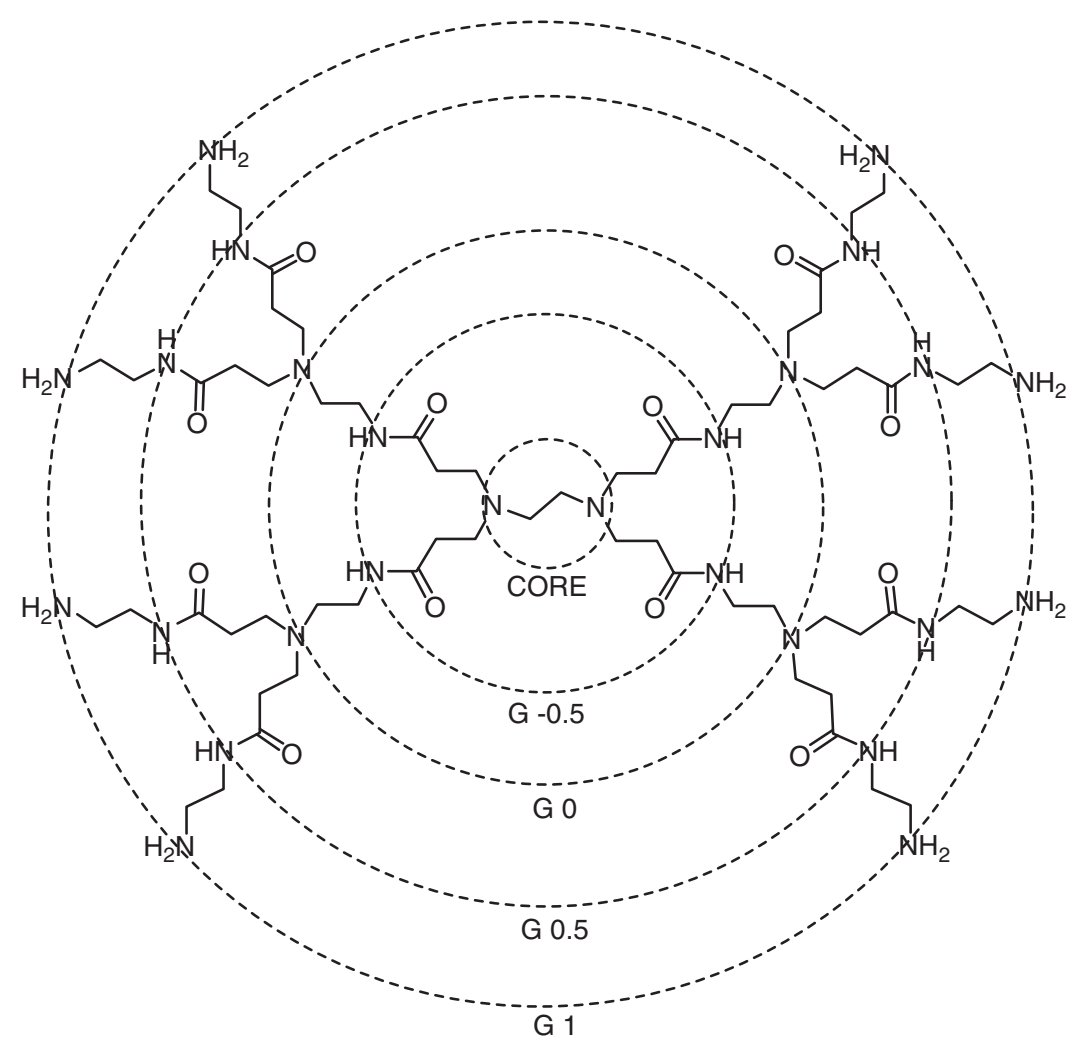

FIGURE 1 | The core-shell architecture of a poly(amidoamine) (PAMAM) dendrimer with an ethylene diamine core with a typical generation numbering scheme. Half-generation PAMAM dendrimers may have carboxyl or methyl ester terminal groups. Unmodified full-generation PAMAM dendrimers have amine surface groups. 


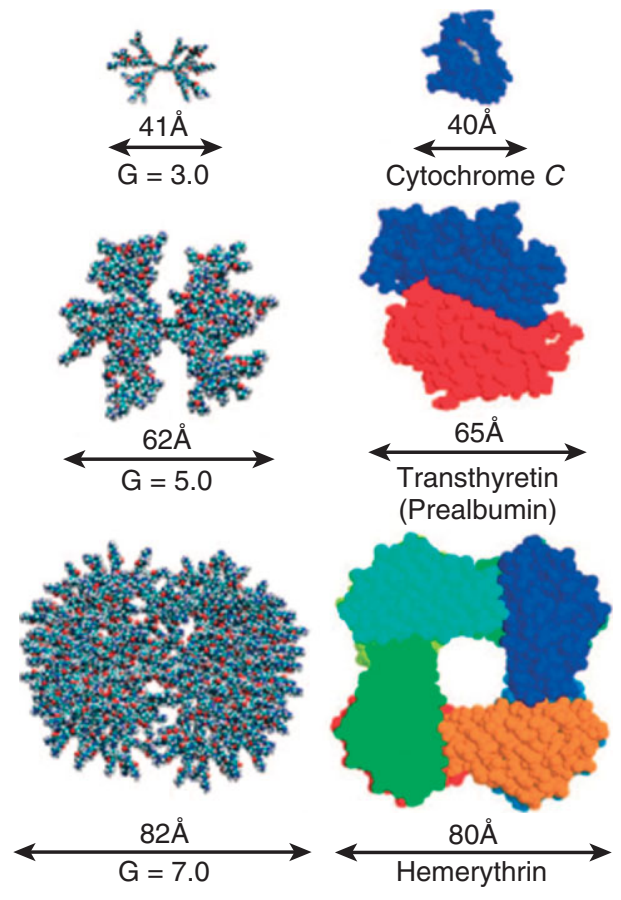

FIGURE 2 | Absolute size comparison of PAMAM dendrimers of varied generation to several key proteins. (Reprinted with permission from Ref 31. Copyright 2007 American Chemical Society).

while being stable enough to remain in circulation. The vast majority of the dendrimers synthesized and published in literature are insoluble in physiological conditions or are incapable of remaining soluble after the addition of functional molecules and are inappropriate for biological applications. However, several classes of dendrimers have been shown to be useful scaffolds for biomedical applications; examples include polyesters, ${ }^{19,20}$ polylysine,${ }^{21}$, and polypropyleneimine (PPI or DAB) dendrimers. ${ }^{22,23}$

The most widely used dendrimers in biomedical applications are poly(amidoamine) (PAMAM) dendrimers. The polyamide backbone synthesized from repeating reactions of methyl acrylate and ethylenediamine helps the macromolecule maintain water solubility and minimizes immunogenicity. ${ }^{24}$ PAMAM dendrimers of different generation also are able to mimic the size and properties of globular proteins readily found in the body (Figure 2). However, PAMAM dendrimers offer several advantages over proteins, as proteins are fragile and can more easily denature because of changes in temperature, light, or $\mathrm{pH} .{ }^{15}$ The amine-terminated surface of full generation PAMAM dendrimers allows for easy surface modification, enabling the platform to carry and solubilize hydrophobic therapeutic molecules, such as methotrexate, ${ }^{16,24-27}$ in physiological conditions. PAMAM dendrimers exhibit little nonspecific toxicity if the surface amines have been neutralized or appropriately modified. ${ }^{10,12,28-31}$

Additional dendrimers have been synthesized to be biodegradeable by incorporating peptide-based backbones that act as substrates for metabolic pathways. ${ }^{32}$ Although these constructs could greatly enhance the prevention of bioaccumulation, they also serve as a reminder to consider potential degradation products of the dendrimer platforms, as they may exhibit an undesired toxicity.

\section{TARGETED TOXICITY}

Targeted delivery of cytotoxic drugs aims to eliminate nonspecific toxicities while increasing local concentrations in specific materials through a number of mechanisms. ${ }^{33}$ Similar to other polymeric materials, dendrimers can target and achieve enhanced specificity via active and passive pathways.

\section{Surface Decoration and Functionalization}

Active targeting uses a molecule, such as an antibody or ligand, to mediate delivery of its payload (drug or otherwise) to cells by binding to cell-specific molecules. Typically, these molecules or delivery vehicles bind through receptors highly expressed on target cells. The interactions between the targeting ligand and cell-surface receptor allow the therapeutic or payload to selectively reach the cancer cells and even be ushered inside via receptor-mediated processes. ${ }^{1}$

The multivalent effect associated with the display of multiple binding ligands on the dendrimer surface enhances the uptake of the dendritic scaffold compared to single ligands. ${ }^{14}$ Multivalent interactions, caused by the simultaneous binding of multiple ligands, allow for the dendrimers to increase the binding avidities of the platform, even when individual ligands have low affinities for the targeted receptor receptor. The PAMAM platform (Figure 3) has been successfully used as a scaffold for the attachment of multivalent targeting molecules including antibodies, ${ }^{34-38}$ peptides, ${ }^{39,40}$ T-antigens, ${ }^{41-43}$, and folic acid. ${ }^{16,24,25,44-50}$ The targeting ligands anchor the dendrimers to locations where specific receptors are expressed on cell surfaces. Hong showed the avidity effects of dendrimers with an increasing amount of conjugated folic acid. ${ }^{14}$ The surface plasmon resonance data showed a linear increase in on-rate and exponential decrease in off-rate of the targeted dendrimer for increasing amount of folic acid per dendrimer. The increased avidity allows targeted dendrimer-drug conjugates to deliver a higher dose 


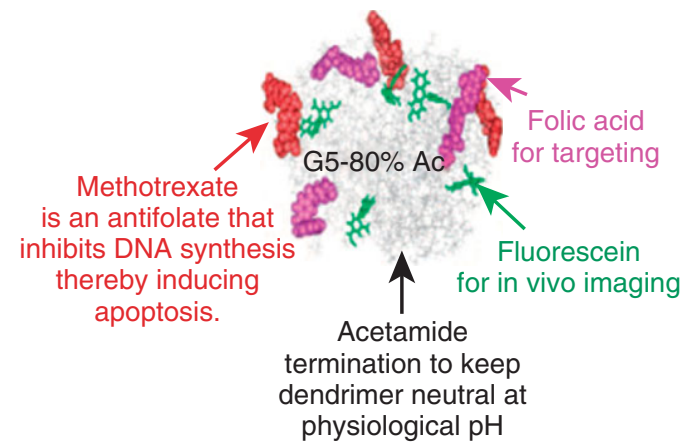

FIGURE 3 | Schematic of a multifunctional targeted nanodevice based on the PAMAM dendrimer scaffold. (Reprinted with permission from Ref 31. Copyright 2007 American Chemical Society).

specifically to targeted cells while avoiding normal cells, thus avoiding the systemic toxicity of current therapeutics.

In 2002, a Quintana et al. ${ }^{51}$ demonstrated mediated internalization of folic acid functionalized G5 PAMAM dendrimers by folate receptors on carcinoma KB cells. This study demonstrated a 100 -fold improvement in cytotoxicity of targeted dendrimers with methotrexate compared to free methotrexate. In 2004, Thomas et al. ${ }^{36}$ demonstrated successful targeting of prostate specific membrane antigens by antibody-conjugated dendrimers. The following year, a second study by Thomas et al. ${ }^{46}$ demonstrated internalization of a folic acid targeted dendrimers carrying methotrexate. Results from this study indicated internalization was through folic acid receptor-mediated endocytosis, suggesting that the delivery devices might be able to overcome methotrexate induced drug resistance.

Kukowska-Latallo et al. ${ }^{16}$ demonstrated the effectiveness of the multifunctional folic acid, methotrexate-conjugated dendrimer in vivo. Conducted in SCID mice bearing KB tumors for 99 days,

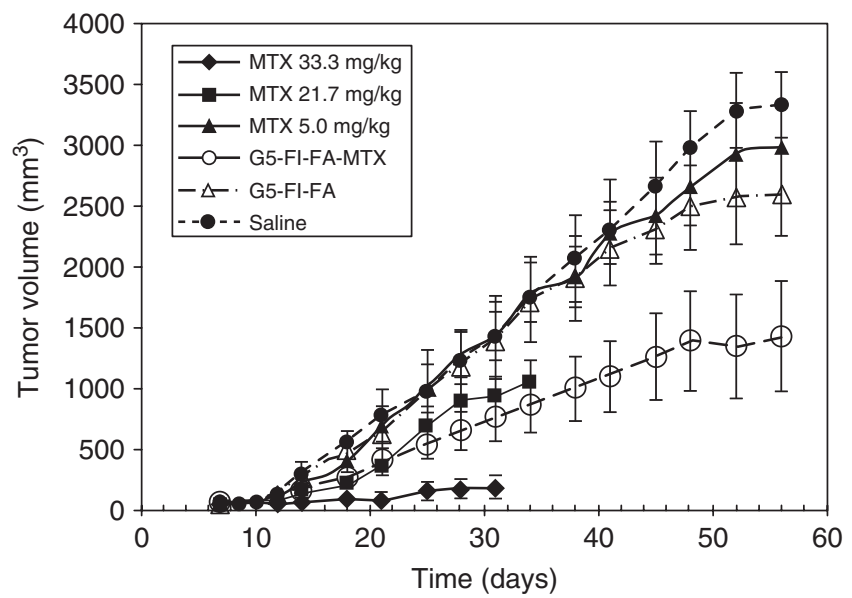

biweekly injections of the multifunctional dendrimer delivery device caused a significant increase in the survival rate compared to treatment with free methotrexate. The conjugate showed 10 -fold higher efficacy compared to the equivalent dose of free drug (Figure 4).

Although dendrimers generally offer an increased synthetic control and monodispersity over other polymeric platforms, understanding conjugate product distributions is still a critical area of interest shared over all multivalent or multifunctional materials. Functionalized dendrimers are typically reported to have an average number of targeting ligands or therapeutics, where the distribution of the population cannot be distinguished by most common characterization techniques including NMR, GPC, and MALDI. ${ }^{24}$ The distributions become increasingly complicated as additional functionalities are conjugated to the dendrimer, though it should be noted that the polydispersity of these multifunctional dendrimers remain lower than other common polymeric platforms. Minute changes in reaction conditions between batches can significantly alter these distributions and as a result affect the biological activity, including toxicity, of the material. It is possible synthetic errors or alterations can create portions of the product distribution that are nonactive or, worse, nonspecifically toxic. Because of this, it would be highly desirable if structure-function studies could be performed on the individual populations within the distribution to determine which populations have the desirable biological activities. Unfortunately, no protocol for separating these populations has been published. New developments in dendrimer synthesis aim to bypass issues of distributions completely through orthogonal coupling chemistry. ${ }^{52}$ One might imagine that future dendritic platforms having enhanced monodispersity with an optimal number of targeting and therapeutic ligands for any given

FIG URE 4 | Tumor growth in severely combined immunodeficient (SCID) mice bearing KB xenografts during treatment with tri-functional $\mathrm{G} 5$ dendrimer with folic acid (FA), fluorescein $(\mathrm{FI})$, and methotrexate (MTX). The dose of the conjugate $(55.0 \mathrm{mg} / \mathrm{kg})$ was equivalent to the lowest dose of free methotrexate $(5.0 \mathrm{mg} / \mathrm{kg})$ is as effective in tumor growth delay as the intermediated dose of free methotrexate $(21.7 \mathrm{mg} / \mathrm{kg}){ }^{16}$ 
application. Regardless, until dendrimers with exact numbers of ligands be separated or synthesized, the distribution of the ligands between batches must be consistent. Otherwise, it will be difficult for dendrimers to be a viable option for many therapeutic applications.

\section{Enhanced Permeation and Retention Effect}

In addition to ligand/receptor targeting, dendrimers have also been reported to use passive approaches that exploit the size relationship between large macromolecules and the pore size in tumor tissue. This is called the enhanced permeation and retention effect (EPR) and is often seen in cancerous tissues as compared with healthy cells. ${ }^{53-55}$ The defective architecture and decreased lymphatic drainage present in tumor tissues result in the macromolecular complex to be retained within the tumor. This is usually a less effective approach particularly in smaller tumors where the vasculature is not 'leaky'. Malik successfully showed that PAMAM dendrimers conjugated with the hydrophobic anticancer drug, cisplatin, selectively accumulated in B16F10 tumor-bearing mice in 50fold greater quantities compared to free drug. ${ }^{56}$ Decreased systemic toxicity and increased solubility were reported for the conjugated cisplatin. Bhadra used PEGylated G4 PAMAM dendrimers for carriers of anticancer drug 5-fluorouracil. ${ }^{57}$ The platforms were injected intravenously into the caudal vein of rats and the blood level of drug was measured. The drug level of 5-fluorouracil in PEGylated samples was enhanced, and detectable up to $12 \mathrm{~h}$ after the drug was injected.

Enhanced retention of dendrimers has also been shown in inflamed tissues. Chauhan complexed the anti-inflammatory drug indomethacin to G4 PAMAMs and injected intravenously to arthritic rats. ${ }^{58}$ The indomethacin concentrations in inflamed joints were 2.29 times greater in complexed dendrimer treated animals compared to animals treated with free drug. The arthritic joint contained lymphatic drainage, but the complex still had an extended retention at the inflamed site. Asthana reported similar results in vitro and in vivo in inflammatory models with antiinflammatory drug flurbiprofen/PAMAM dendrimer formulations. ${ }^{59}$

\section{Minimizing Nonspecific Toxicities}

In addition to enhancing desired specific toxicities of dendrimer platforms for applications such as cancer therapy, it is crucial to understand and minimize nonspecific interactions with healthy cells. The dendrimer periphery can significantly alter the cytotoxicity of the platform. Similar to other polymeric materials, cationic dendrimer surface charges interact with negatively charged biological surface to lead to structural disruptions. ${ }^{60}$ Modification of the dendrimer can minimize these effects. Larger generation dendrimers typically display a smaller influence from their well-protected cores as charges on their arms can be shielded by backfolding and steric effects.

In general, cationic dendrimers have been shown to exhibit nonspecific toxicities (Figure 5) and must be modified to prevent accumulation in the liver. ${ }^{61,62}$ Roberts showed that cationic PAMAM dendrimers were toxic at low concentrations in V79 Chinese hamster lung fibroblasts. ${ }^{63}$ Ninety percent cell death was observed using MTT assays at $1 \mathrm{nM}$ for generation 3, $10 \mu \mathrm{M}$ for generation 5 , and $100 \mathrm{nM}$ for generation 7 . The same group observed similar toxicities for cationic PAMAM, DAB, and diaminoethane dendrimers in B16F10 murine melanoma, corticotropinreleasing factor (CRF) human lymphoblastic leukemia and HepG2 human hepatoma. ${ }^{61}$ Cationic PAMAM dendrimers were less toxic than $\mathrm{DAB}$ dendrimers of equal surface charge.

PAMAM dendrimers with increasing charge densities have shown increasing toxicities in Caco-2 cells. ${ }^{62}$ It was shown that the cytotoxicity of amineterminated dendrimers was significantly higher when compared to anionic half-generation dendrimers at concentrations across the micromolar range. The cytotoxicity of each type of dendrimer increased with size and concentration. Cytoxicity was significantly decreased (IC50 approximately $0.13 \mathrm{mM}$ to greater than $1 \mathrm{mM}$ ) when the cationic dendrimer surface was modified with six lauroyl or four PEG chains, which likely shielded the positive charge of the surface.

Quintana in $2002^{51}$ and Nigavekar in $2004^{64}$ showed that by neutralizing the surface amines of PAMAM dendrimers with acetyl groups, nonspecific toxicities and uptake could be minimized. The acetyl capping of the dendrimer also allows for increased clearance from the body, minimizing effects from longterm treatment. Further evidence that PEGlyation of amino-terminated PAMAM dendrimers reduces immunogenicity and increases solubility was shown by Kobayashi. ${ }^{65}$ The PEG terminated dendrimer increased the lifetime in the blood stream compared to the cationic parent material. Hydroxyl and methyoxyl terminated polyester dendrimers were also shown to be nontoxic in vivo up at high concentrations up to $40 \mathrm{mg} / \mathrm{kg}$. ${ }^{19,66}$ The differences in toxicities between cationic and anionic dendrimers have also been confirmed in vivo. ${ }^{67}$ Using a zebrafish embryo model, carboxyl terminated dendrimer was significantly less 
(a) Control

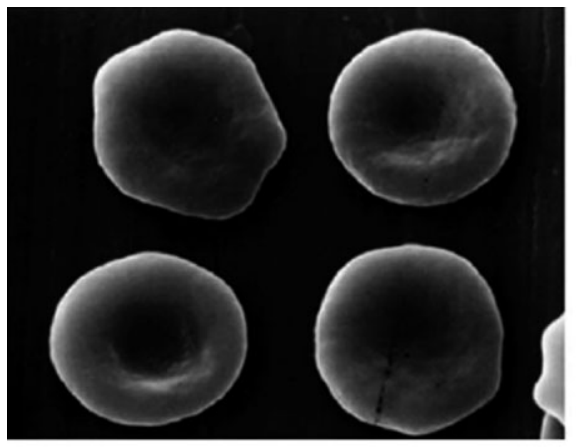

(c) PAMAM gen $4 ; 1 \mathrm{mg} / \mathrm{mL}$

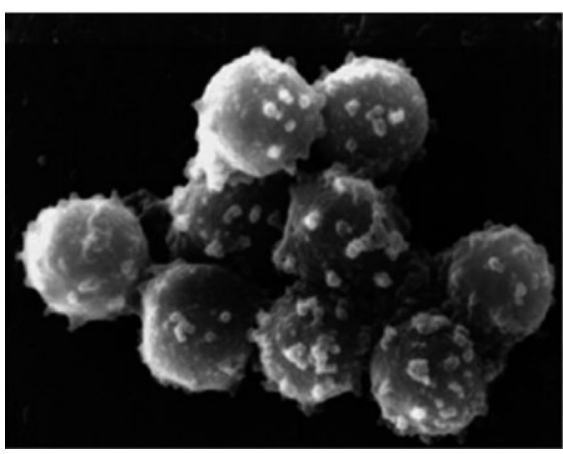

(b) PAMAM gen 4; $10 \mu \mathrm{g} / \mathrm{mL}$

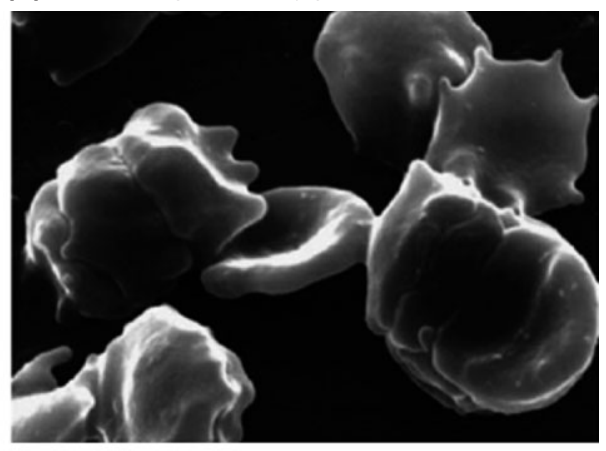

(d) PAMAM gen $3.5 ; 1 \mathrm{mg} / \mathrm{mL}$

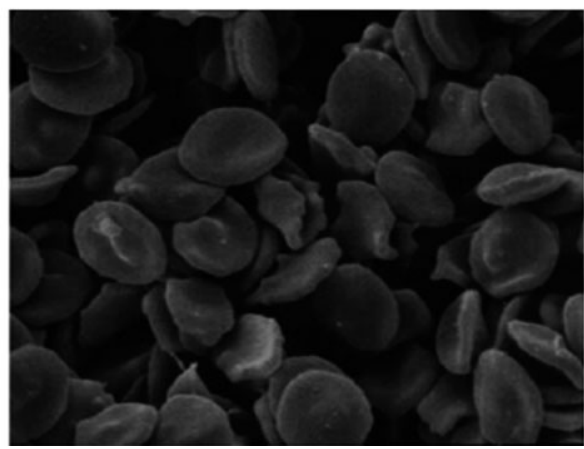

FIGURE 5 | Scanning electron microscopy of red blood cell (RBC) incubated with poly(amidoamine) (PAMAM) dendrimers for $1 \mathrm{~h}$. The cationic dendrimer (generation 4) disrupts the structure the integrity of the cell structure at low concentrations. (Reprinted with permission from Ref 61. Copyright 2000 Elsevier).
Dendrimer exposure (6-120 hpf)

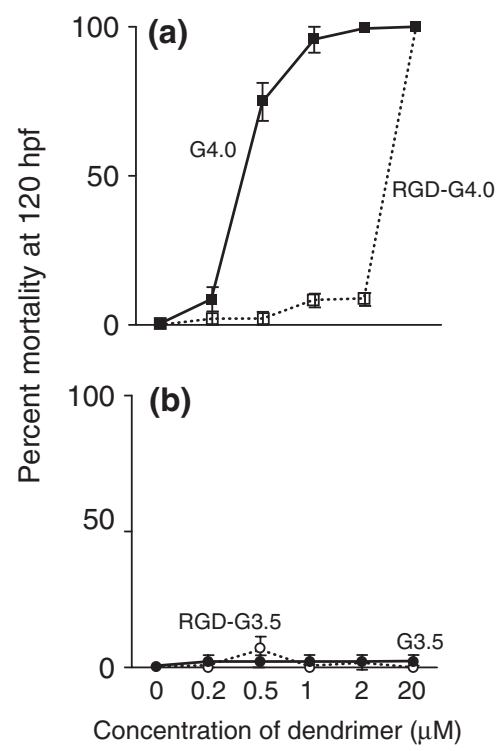

FIG URE 6 | Effect of net charge on amine-terminated G4 and carboxylic acid-terminated G3.5 dendrimer-induced mortality in the zebrafish embryo. (A) Mortality assessed at $120 \mathrm{~h}$ post-fertilization for embryos exposed to G4 or RGD-G4 dendrimers from 6 to $120 \mathrm{~h}$ post-fertilization. (B) Mortality evaluated at $120 \mathrm{~h}$ post-fertilization for embryos exposed to G3.5 or RGD-G3.5 dendrimers from 6 to $120 \mathrm{~h}$ post-fertilization. (Reprinted with permission from Ref 67 . Copyright 2007 Elsevier). toxic than G4 amine-terminated dendrimer (Figure 6). In the same study, surface modification with RGD also reduced toxicity.

The mechanism behind nonspecific binding of cationic dendrimers has been explored by Hong and Leroueil. ${ }^{29,30,60}$ They have shown that positively charged polymeric material, including amine terminated PAMAM dendrimers, causes the formation of holes in cell membranes resulting in nonspecific internalization of material (Figure 7). Studies were performed on dimyristoylphosphatidylcholine (DMPC) lipid bilayers atomic force microscopy and on $\mathrm{KB}$ and Rat 2 cells in culture, and this was evaluated by LDH release assays. Generation 7 PAMAM dendrimers were observed to form holes $(15-40 \mathrm{~nm}$ in diameter) in DMPC while generation 5 dendrimers expended existing holes. Experimental results suggest that the dendrimer is also more efficient at increasing membrane permeability compared to a linear polymer of the same charge density. Neither hole formation nor internalization was observed for charge neutral, nontargeted dendrimers.

Further understanding the toxicity of cationic dendrimers is crucial for successful use in gene transfection applications, ${ }^{8,68-71}$ where a positively charged surface is required for ionic complexation with DNA and to act as a proton buffer, leading to endosome disruption. ${ }^{71}$ Although dendrimer polyplexes have shown effective transfection and lower toxicities 
(a)
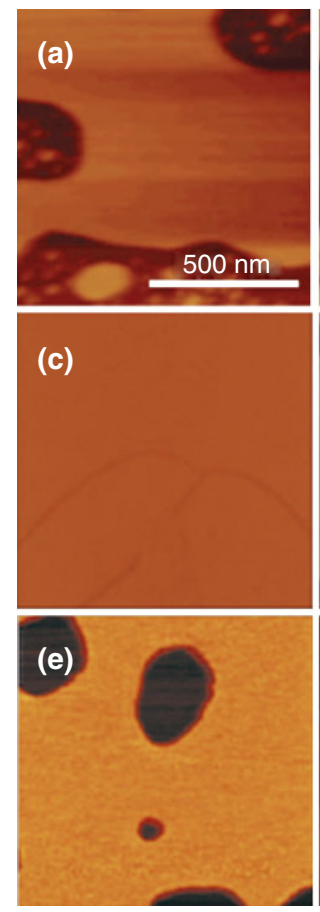

(b)
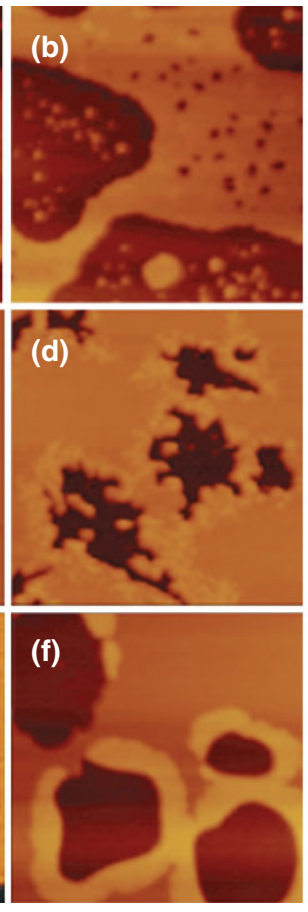

(a) $\mathrm{G} 7-\mathrm{NH}_{2}$

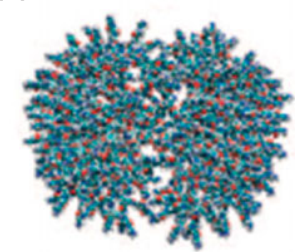

(b) $\mathrm{G} 5-\mathrm{NH}_{2}$

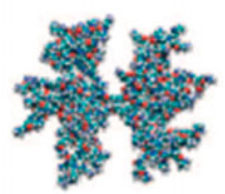

(c) G5-Ac

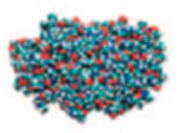

(c)

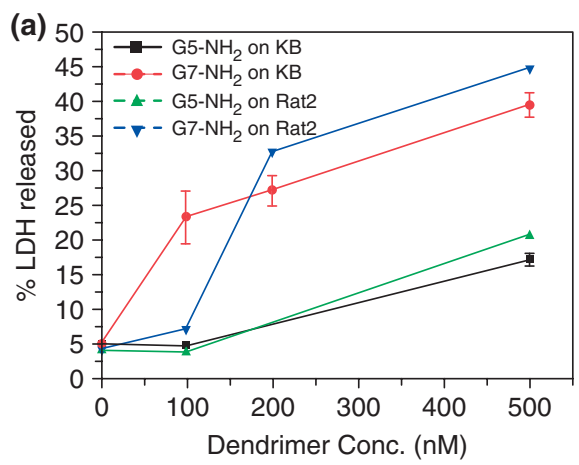

(b)

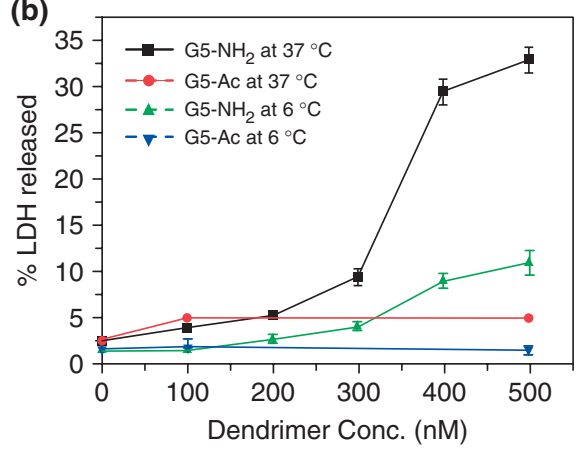

FIG URE 7 | Poly(amidoamine) (PAMAM) dendrimer interactions with biological membranes. (I) Atomic force microscopy (AFM) observation of dimyristoylphosphatidylcholine (DMPC) supported lipid bilayers (a), (c), and (e) before and after incubation with (b) amine-terminated G7 (G7- $\mathrm{NH}_{2}$ ), (d) amine-terminated G5 (G5- $\mathrm{NH}_{2}$ ), and (f) acetylated G5 (G5-Ac) PAMAM dendrimers, respectively. (II) Space-filling models of chemical structures of (a) G7- $\mathrm{NH}_{2}$, (b) G5- $\mathrm{NH}_{2}$, and (c) G5-Ac PAMAM dendrimers. (III) Lactate dehydrogenase (LDH) leakage as a result of cell exposure to PAMAM dendrimers. (a) Size effect of G7- $\mathrm{NH}_{2}$ and G5- $\mathrm{NH}_{2}$ on the LDH leakage out of $\mathrm{KB}$ and Rat2 cells after incubation at $37^{\circ} \mathrm{C}$ for $3 \mathrm{~h}$ and (b) surface group dependency on the LDH leakage at different temperatures. Note that larger dendrimers $\left(\mathrm{G}^{-}-\mathrm{NH}_{2}\right)$ induce formation of new nanoscale holes in the bilayers as seen in the AFM images and cause more amount of LDH leakage out of live cells than $\mathrm{G} 5-\mathrm{NH}_{2}$. G5- $\mathrm{NH}_{2}$ dendrimers do not cause new hole formation in the lipid bilayers but instead expand preexisting defects. In contrast, G5-Ac dendrimers do not cause hole formation, expansion of preexisting defects, or LDH leakage out of live cells. (Reprinted with permission from Ref 31. Copyright 2007 American Chemical Society).

compared to other polymeric transfection agents in vitro, further work optimizing toxicity levels must be performed to translate these devices to clinical situations.

In addition to serving as a delivery platform, neutralized or modified dendrimers are being explored as stable, biocompatible, water-soluble coating for other devices. Acetylated G5 PAMAM dendrimers have been attached to carbon nanotubes ${ }^{72}$ for targeted delivery and to iron oxide nanoparticles ${ }^{50}$ for MRI. These show that the use of dendrimers can be expanded to enhance the biomedical applicability of other platforms as well.

\section{Biodistribution}

Multiple studies have been performed evaluating the biodistribution of dendrimers using 125Iradiolabeling, ${ }^{61,73}$ gadolinium labeling, ${ }^{74} 3 \mathrm{H}^{64}$ or 14C labeling. ${ }^{63}$ Similar to the effects surface modifications can have on the toxicity, these labeling techniques may alter the distribution of the platform. However, general conclusions as to the fate of administered dendrimer can be made. Charged and hydrophobic dendrimers are rapidly cleared from the circulation, typically by the liver. Hydrophilic dendrimers with hydroxyl or PEG surfaces have extended circulation lifetimes. Larger dendrimers delay renal elimination compared to smaller platforms. Nigavekar showed that net surface charge of generation 5 PAMAM dendrimers modifies the biodistribution. ${ }^{64}$ Amine terminated PAMAMs deposition into tissues is higher than acetylated dendrimers, though the distribution is similar. Highest levels were found in the lungs, liver, and kidney. Approximately $50 \%$ of the acetyl capped PAMAMs and approximately $30 \%$ of the amine-terminated PAMAMs were excreted via urine over 7 days, approximately $25 \%$ and approximately $8 \%$ during the first day, respectively.

The biodistribution of targeted systems has also been studied. In folic acid targeted PAMAMs, 
Kukowska-Latallo et al. showed that levels of the radiolabeled conjugate increased in tissues expressing high levels of the folate receptor, the liver, kidney and tumor, compared with nontargeted dendrimer. ${ }^{16}$ The nontargeted dendrimer dropped in concentration significantly $2 \mathrm{~h}$ after injection in these tissues. The differences in accumulation, distribution, and excretion supported the preferential uptake model based on folic acid receptor levels present on the cell membranes.

Yang et al. evaluated the distribution epidermal growth factor (EGF) targeting boronated PAMAM dendrimers. ${ }^{75}$ Rats bearing F98 $8_{\mathrm{EGFR}}$ gliomas and F98 WT (wild-type) receptor negative tumors were used in the study. Twenty-four hours after administration, $47.4 \%$ of the dendrimer dose was located in the receptor positive gliomas compared with $12.3 \%$ in the wild-type tumor. The mean survival time of rats receiving the conjugate was 53 days, and only 31 days for the control.

Although enhanced uptake in target cells has clearly been demonstrated, the relative accumulation of targeted dendrimers in target tissue versus healthy tissue must be taken into account. The use of cell markers that are expressed in both target and healthy tissue as targets can lead to potentially toxic accumulations of drug or dendrimer within the healthy tissue. Although in some cases this risk may be acceptable, extensive studies must be completed documenting that the targeted treatment is more effective than systemic or no treatment. With future developments and as more cell-specific markers are found, the risks of harming healthy tissue with targeted dendrimers will diminish.

\section{CONCLUSION}

Dendrimers have been used for multiple targeting, imaging, and delivery applications. These highly customizable devices can be engineered to display an appropriate amount of specificity through interactions between conjugated ligands and cellular receptors and enhanced retention in abnormal tissue architecture, while minimizing nonspecific uptake and toxicities by neutralizing surfaces charges. Considerations including solubility of the platform, coupling chemistry required, and required number of ligands needed must be evaluated when determining the necessary chemical backbone and generation of dendrimer. Dendrimers can enhance the toxicity of a material by increasing the local concentration of a therapeutic via multivalent interactions with surface-bound ligands and by enhancing retention within the targeted tissue. Nonspecific toxicities have been shown for positively charged dendrimers, so care must be shown to appropriately neutralize the dendritic device.

It has been shown that certain dendrimers, such as those with the PAMAM backbone and neutralized surfaces groups, are appropriate for many biological applications. PAMAM dendrimers expressing various targeting groups have successfully been used to target therapeutic and sensing molecules while maintaining an appropriate level of biocompatibility. The multifunctional PAMAM dendrimer conjugated with folic acid and methotrexate will soon enter phase I trials.

For the platform to reach further into the mainstream, progress must be made in the synthesis and characterization of the dendrimer platform and its conjugates. Recent work showing the ability to better understand and isolate product distributions in dendrimer conjugates has the potential to facilitate an increased awareness of the impact these materials have on biological systems. By optimizing the product distribution, the dendrimer platform can offer an even greater degree of control and customization over other polymeric delivery devices.

There remains a need to perform appropriate toxicology studies for dendrimer therapeutics. The properties of functionalized or modified dendrimer can be significantly different from the parent dendrimer. It would be inappropriate to draw conclusions on the toxicity of a functionalized dendrimer based on the general properties of the scaffold. Effective dendrimer platforms will succeed in clinical trials only after specific toxicity testing has been performed on appropriate animal models. Computer-aided design can successfully create a biocompatible dendrimer structures that exploit the advantages of the platform.

\section{REFERENCES}

1. Allen TM. Ligand-targeted therapeutics in anticancer therapy. Nat Rev Cancer 2002, 2:750-763.

2. Liu Y, Miyoshi H, Nakamura M. Nanomedicine for drug delivery and imaging: a promising avenue for cancer therapy and diagnosis using targeted functional nanoparticles. Int J Cancer 2007, 120:2527-2537.

3. Boas U, Heegaard PM. Dendrimers in drug research. Chem Soc Rev 2004, 33:43-63. 
4. Agarwal A, Saraf S, Asthana A, Gupta U, Gajbhiye $\mathrm{V}$, et al. Ligand based dendritic systems for tumor targeting. Int J Pharm 2008, 350:3-13.

5. Agarwal A, Asthana A, Gupta U, Jain NK. Tumour and dendrimers: a review on drug delivery aspects. J Pharm Pharmacol 2008, 60:671-688.

6. Cloninger MJ. Biological applications of dendrimers. Curr Opin Chem Biol 2002, 6:742-748.

7. Cheng Y, Xu Z, Ma M, Xu T. Dendrimers as drug carriers: applications in different routes of drug administration. J Pharm Sci 2008, 97:123-143.

8. Dufès C, Uchegbu IF, Schätzlein AG. Dendrimers in gene delivery. Adv Drug Deliv Rev 2005, 57:2177-2202.

9. Gillies ER, Frechet JMJ. Dendrimers and dendritic polymers in drug delivery. Drug Discov Today 2005, 10:35-43.

10. Lee CC, MacKay JA, Frechet JMJ, Szoka FC. Designing dendrimers for biological applications. Nat Biotechnol 2005, 23:1517-1526.

11. Liu M, Frechet JM. Designing dendrimers for drug delivery. Pharm Sci Technol Today 1999, 2:393-401.

12. Svenson S, Tomalia DA. Dendrimers in biomedical applications-reflections on the field. Adv Drug Deliv Rev 2005, 57:2106-2129.

13. Svenson S. Dendrimers as versatile platform in drug delivery applications. Eur J Pharm Biopharm 2009, 71:445-462.

14. Hong S, Leroueil PR, Majoros IJ, Orr BG, Baker JR $\mathrm{Jr}$, et al. The binding avidity of a nanoparticle-based multivalent targeted drug delivery platform. Chem Biol 2007, 14:107-115.

15. Esfand R, Tomalia DA. Poly(amidoamine) (PAMAM) dendrimers: from biomimicry to drug delivery and biomedical applications. Drug Discov Today 2001, $6: 427-436$.

16. Kukowska-Latallo JF, Candido KA, Cao Z, Nigavekar SS, Majoros IJ, et al. Nanoparticle targeting of anticancer drug improves therapeutic response in animal model of human epithelial cancer. Cancer Res 2005, 65:5317-5324.

17. Kong G, Braun RD, Dewhirst MW. Hyperthermia enables tumor-specific nanoparticle delivery: effect of particle size. Cancer Res 2000, 60:4440-4445.

18. Frechet JMJ, Tomalia DA, eds. Dendrimers and Other Dendritic Polymers: Chichester, England: Wiley; 2001.

19. Padilla De Jesus OL, Ihre HR, Gagne L, Frechet JM, Szoka FC Jr. Polyester dendritic systems for drug delivery applications: in vitro and in vivo evaluation. Bioconjug Chem 2002, 13:453-461.

20. Morgan MT, Carnahan MA, Immoos CE, Ribeiro AA, Finkelstein S, et al. Dendritic molecular capsules for hydrophobic compounds. J Am Chem Soc 2003, 125:15485-15489.
21. Bourne N, Stanberry LR, Kern ER, Holan G, Matthews $B$, et al. Dendrimers, a new class of candidate topical microbicides with activity against herpes simplex virus infection. Antimicrob Agents Chemother 2000, $44: 2471-2474$.

22. Hollins AJ, Benboubetra M, Omidi Y, Zinselmeyer BH, Schatzlein AG, et al. Evaluation of generation 2 and 3 poly(propylenimine) dendrimers for the potential cellular delivery of antisense oligonucleotides targeting the epidermal growth factor receptor. Pharm Res 2004, 21:458-466.

23. Wang SJ, Brechbiel M, Wiener EC. Characteristics of a new MRI contrast agent prepared from polypropyleneimine dendrimers, generation 2. Invest Radiol 2003, 38:662-668.

24. Majoros IJ, Myc A, Thomas T, Mehta CB, Baker JR Jr. PAMAM dendrimer-based multifunctional conjugate for cancer therapy: synthesis, characterization, and functionality. Biomacromolecules 2006, 7:572-579.

25. Majoros IJ, Thomas TP, Mehta CB, Baker JR Jr. Poly(amidoamine) dendrimer-based multifunctional engineered nanodevice for cancer therapy. J Med Chem 2005, 48:5892-5899.

26. Patri AK, Kukowska-Latallo JF, Baker J, James R. Targeted drug delivery with dendrimers: comparison of the release kinetics of covalently conjugated drug and non-covalent drug inclusion complex. Adv Drug Deliv Rev 2005, 57:2203-2214.

27. Patri AK, Majoros IJ, Baker JR. Dendritic polymer macromolecular carriers for drug delivery. Curr Opin Chem Biol 2002, 6:466-471.

28. Majoros IJ, Keszler B, Woehler S, Bull T, Baker JR. Acetylation of poly(amidoamine) dendrimers. Macromolecules 2003, 36:5526-5529.

29. Hong SP, Bielinska AU, Mecke A, Keszler B, Beals $\mathrm{JL}$, et al. Interaction of poly(amidoamine) dendrimers with supported lipid bilayers and cells: hole formation and the relation to transport. Bioconjug Chem 2004, 15:774-782.

30. Hong SP, Leroueil PR, Janus EK, Peters JL, Kober $\mathrm{MM}$, et al. Interaction of polycationic polymers with supported lipid bilayers and cells: nanoscale hole formation and enhanced membrane permeability. Bioconjug Chem 2006, 17:728-734.

31. Leroueil PR, Hong SY, Mecke A, Baker JR, Orr BG, et al. Nanoparticle interaction with biological membranes: does nanotechnology present a janus face? Acc Chem Res 2007, 40:335-342.

32. Goodwin AP, Lam SS, Frechet JM. Rapid, efficient synthesis of heterobifunctional biodegradable dendrimers. J Am Chem Soc 2007, 129:6994-6995.

33. Backer MV, Gaynutdinov TI, Patel V, Bandyopadhyaya AK, Thirumamagal BTS, et al. Vascular endothelial growth factor selectively targets boronated dendrimers to tumor vasculature. Mol Cancer Ther 2005, 4:1423-1429. 
34. Patri AK, Myc A, Beals J, Thomas TP, Bander NH, et al. Synthesis and in vitro testing of J591 antibodydendrimer conjugates for targeted prostate cancer therapy. Bioconjug Chem 2004, 15:1174-1181.

35. Shukla R, Thomas TP, Peters JL, Desai AM, KukowskaLatallo J, et al. HER2 specific tumor targeting with dendrimer conjugated anti-HER2 mAb. Bioconjug Chem 2006, 17:1109-1115.

36. Thomas TP, Patri AK, Myc A, Myaing MT, Ye JY, et al. In vitro targeting of synthesized antibody-conjugated dendrimer nanoparticles. Biomacromolecules 2004, 5:2269-2274.

37. Wu G, Barth RF, Yang WL, Chatterjee M, Tjarks $\mathrm{W}$, et al. Site-specific conjugation of boron-containing dendrimers to anti-EGF receptor monoclonal antibody cetuximab (IMC-C225) and its evaluation as a potential delivery agent for neutron capture therapy. Bioconjug Chem 2004, 15:185-194.

38. Wu G, Barth RF, Yang WL, Kawabata S, Zhang LW, et al. Targeted delivery of methotrexate to epidermal growth factor receptor-positive brain tumors by means of cetuximab (IMC-C225) dendrimer bioconjugates. Mol Cancer Ther 2006, 5:52-59.

39. Shukla R, Thomas TP, Peters J, Kotlyar A, Myc A, et al. Tumor angiogenic vasculature targeting with PAMAM dendrimer-RGD conjugates. Chem Commun (Camb) 2005, 46:5739-5741.

40. Lesniak WG, Kariapper MS, Nair BM, Tan W, Hutson A, et al. Synthesis and characterization of PAMAM dendrimer-based multifunctional nanodevices for targeting alphavbeta3 integrins. Bioconjug Chem 2007, 18:1148-1154.

41. Sheng KC, Kalkanidis M, Pouniotis DS, Esparon S, Tang CK, et al. Delivery of antigen using a novel mannosylated dendrimer potentiates immunogenicity in vitro and in vivo. Eur J Immunol 2008, 38:424-436.

42. Baek MG, Roy R. Synthesis and protein binding properties of T-antigen containing GlycoPAMAM dendrimers. Bioorg Med Chem 2002, 10:11-17.

43. Taite LJ, West JL. Poly(ethylene glycol)-lysine dendrimers for targeted delivery of nitric oxide. J Biomater Sci Polym Ed 2006, 17:1159-1172.

44. Kono K, Liu M, Frechet JM. Design of dendritic macromolecules containing folate or methotrexate residues. Bioconjug Chem 1999, 10:1115-1121.

45. Shukla S, Wu G, Chatterjee M, Yang W, Sekido M, et al. Synthesis and biological evaluation of folate receptor-targeted boronated PAMAM dendrimers as potential agents for neutron capture therapy. Bioconjug Chem 2003, 14:158-167.

46. Thomas TP, Majoros IJ, Kotlyar A, Kukowska-Latallo JF, Bielinska A, et al. Targeting and inhibition of cell growth by an engineered dendritic nanodevice. $\mathrm{J} \mathrm{Med}$ Chem 2005, 48:3729-3735.
47. Myc A, Douce TB, Ahuja N, Kotlyar A, KukowskaLatallo J, et al. Preclinical antitumor efficacy evaluation of dendrimer-based methotrexate conjugates. Anticancer Drugs 2008, 19:143-149.

48. Myc A, Majoros IJ, Thomas TP, Baker JR. Dendrimerbased targeted delivery of an apoptotic sensor in cancer cells. Biomacromolecules 2007, 8:13-18.

49. Myc A, Patri AK, Baker JR. Dendrimer-based BH3 conjugate that targets human carcinoma cells. Biomacromolecules 2007, 8:2986-2989.

50. Landmark KJ, DiMaggio S, Ward J, Kelly C, Vogt S, et al. Synthesis, characterization, and in vitro testing of superparamagnetic iron oxide nanoparticles targeted using folic acid-conjugated dendrimers. ACS Nano 2008, 2:773-783.

51. Quintana A, Raczka E, Piehler L, Lee I, Myc A, et al. Design and function of a dendrimer-based therapeutic nanodevice targeted to tumor cells through the folate receptor. Pharm Res 2002, 19:1310-1316.

52. Goyal P, Yoon K, Weck M. Multifunctionalization of dendrimers through orthogonal transformations. Chemistry 2007, 13:8801-8810.

53. Modi S, Prakash Jain J, Domb AJ, Kumar N. Exploiting EPR in polymer drug conjugate delivery for tumor targeting. Curr Pharm Des 2006, 12:4785-4796.

54. Jain RK. Delivery of molecular medicine to solid tumors: lessons from in vivo imaging of gene expression and function. J Control Release 2001, 74:7-25.

55. Jain RK. Transport of molecules, particles, and cells in solid tumors. Annu Rev Biomed Eng 1999, 1:241-263.

56. Malik N, Evagorou EG, Duncan R. Dendrimerplatinate: a novel approach to cancer chemotherapy. Anticancer Drugs 1999, 10:767-776.

57. Bhadra D, Bhadra S, Jain S, Jain NK. A PEGylated dendritic nanoparticulate carrier of fluorouracil. Int J Pharm 2003, 257:111-124.

58. Chauhan AS, Jain NK, Diwan PV, Khopade AJ. Solubility enhancement of indomethacin with poly(amidoamine) dendrimers and targeting to inflammatory regions of arthritic rats. J Drug Target 2004, 12:575-583.

59. Asthana A, Chauhan AS, Diwan PV, Jain NK. Poly(amidoamine) (PAMAM) dendritic nanostructures for controlled site-specific delivery of acidic antiinflammatory active ingredient. AAPS PharmSciTech 2005, 6:E536-E542.

60. Leroueil PR, Berry SA, Duthie K, Han G, Rotello VM, et al. Wide varieties of cationic nanoparticles induce defects in supported lipid bilayers. Nano Lett 2008, 8:420-424.

61. Malik N, Wiwattanapatapee R, Klopsch R, Lorenz K, Frey $\mathrm{H}$, et al. Dendrimers: relationship between structure and biocompatibility in vitro, and preliminary 
studies on the biodistribution of 125I-labelled polyamidoamine dendrimers in vivo. J Control Release 2000, 65:133-148.

62. Jevprasesphant R, Penny J, Jalal R, Attwood D, McKeown NB, et al. The influence of surface modification on the cytotoxicity of PAMAM dendrimers. Int J Pharm 2003, 252:263-266.

63. Roberts JC, Bhalgat MK, Zera RT. Preliminary biological evaluation of polyamidoamine (PAMAM) starburst dendrimers. J Biomed Mater Res 1996, 30:53-65.

64. Nigavekar SS, Sung LY, Llanes M, El-Jawahri A, Lawrence TS, et al. $3 \mathrm{H}$ dendrimer nanoparticle organ/tumor distribution. Pharm Res 2004, 21:476-483.

65. Kobayashi H, Kawamoto S, Saga T, Sato N, Hiraga A, et al. Positive effects of polyethylene glycol conjugation to generation-4 polyamidoamine dendrimers as macromolecular MR contrast agents. Magn Reson Med 2001, 46:781-788.

66. Ihre HR, Padilla De Jesus OL, Szoka FC Jr, Frechet JM. Polyester dendritic systems for drug delivery applications: design, synthesis, and characterization. Bioconjug Chem 2002, 13:443-452.

67. Heiden TC, Dengler E, Kao WJ, Heideman W, Peterson RE. Developmental toxicity of low generation PAMAM dendrimers in zebrafish. Toxicol Appl Pharmacol 2007, 225:70-79.

68. Kukowska-Latallo JF, Bielinska AU, Johnson J, Spindler R, Tomalia DA, et al. Efficient transfer of genetic material into mammalian cells using starburst polyamidoamine dendrimers. Proc Natl Acad Sci U S A 1996, 93:4897-4902.
69. Tang MX, Redemann CT, Szoka FC Jr. In vitro gene delivery by degraded polyamidoamine dendrimers. Bioconjug Chem 1996, 7:703-714.

70. Haensler J, Szoka FC Jr. Polyamidoamine cascade polymers mediate efficient transfection of cells in culture. Bioconjug Chem 1993, 4:372-379.

71. Sonawane ND, Szoka FC Jr, Verkman AS. Chloride accumulation and swelling in endosomes enhances DNA transfer by polyamine-DNA polyplexes. $J$ Biol Chem 2003, 278:44826-44831.

72. Shi X, Wang SH, Shen M, Antwerp ME, Chen X, et al. Multifunctional dendrimer-modified multiwalled carbon nanotubes: synthesis, characterization, and in vitro cancer cell targeting and imaging. Biomacromolecules 2009, 10:1744-1750.

73. Wilbur DS, Pathare PM, Hamlin DK, Buhler KR, Vessella RL. Biotin reagents for antibody pretargeting. 3. synthesis, radioiodination, and evaluation of biotinylated starburst dendrimers. Bioconjug Chem 1998, 9:813-825.

74. Langereis S, de Lussanet QG, van Genderen Marcel HP, Backes WH, Meijer EW. Multivalent contrast agents based on Gadolinium-Diethylenetriaminepentaacetic acid-terminated poly(propylene imine) dendrimers for magnetic resonance imaging. Macromolecules 2004, 37:3084-3091.

75. Yang W, Barth RF, Wu G, Bandyopadhyaya AK, Thirumamagal BT, et al. Boronated epidermal growth factor as a delivery agent for neutron capture therapy of EGF receptor positive gliomas. Appl Radiat Isot 2004, 61:981-985. 